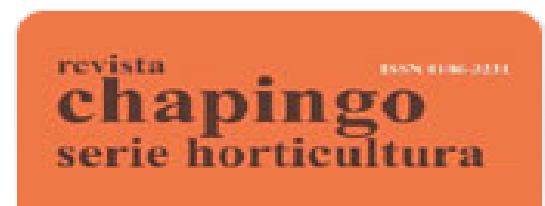

\title{
REVISTA CHAPINGO SERIE HORTICULTURA
}

ISSN: 1027-152X

revistahorticultura29@gmail.com

Universidad Autónoma Chapingo

México

Contreras-Morales, E.; Almaguer-Vargas, G.; Espinoza-Espinoza, J. R.; Maldonado-Torres, R.; Álvarez-Sánchez, E.

DISTRIBUCIÓN RADICAL DE ÁRBOLES DE LIMÓN PERSA (Citrus latifolia Tan.)

REVISTA CHAPINGO SERIE HORTICULTURA, vol. 14, núm. 2, mayo-agosto, 2008, pp. 223-234

Universidad Autónoma Chapingo

Chapingo, México

Disponible en: http://www.redalyc.org/articulo.oa?id=60911556016

- Cómo citar el artículo

Número completo

- Más información del artículo

Página de la revista en redalyc.org

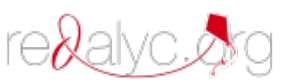

Sistema de Información Científica

Red de Revistas Científicas de América Latina, el Caribe, España y Portugal Proyecto académico sin fines de lucro, desarrollado bajo la iniciativa de acceso abierto 


\title{
DISTRIBUCIÓN RADICAL DE ÁRBOLES DE LIMÓN PERSA (Citrus latifolia Tan.)
}

\author{
E. Contreras-Morales ${ }^{1}$; G. Almaguer-Vargas ${ }^{2 \pi}$; \\ J. R. Espinoza-Espinoza²; R. Maldonado-Torres ${ }^{3}$; \\ E. Álvarez-Sánchez ${ }^{3}$ \\ ${ }^{1}$ Centro de Bachillerato Tecnológico Agropecuario Núm. 135, \\ Arroyo Hondo, Mizantla, Veracruz MÉXICO. \\ ${ }^{2}$ Departamento de Fitotecnia. Universidad Autónoma Chapingo. \\ Km. 38.5 Carretera México-Texcoco, Chapingo, Estado de México. MÉXICO. C. P. 56230. \\ Correo-e: almaguervargas@hotmail.com ("Autor responsable). \\ ${ }^{3}$ Departamento de Suelos. Universidad Autónoma Chapingo. \\ Km. 38.5 Carretera México-Texcoco, Chapingo, Estado de México. MÉXICO. C. P. 56230.
}

\section{RESUMEN}

\begin{abstract}
Este estudio fue hecho para conocer la distribución radical de árboles de limón persa cultivados en un suelo arenoso y uno arcilloso en Martínez de la Torre, Ver. Se empleó el método de la pared del perfil del suelo, clasificando las raíces en cuatro categorías de acuerdo con su diámetro: R1 (>15 mm), R2 (5-15 mm), R3 (1-5 mm) y R4 (<1 mm). Las dimensiones de la cepa fueron $4 \mathrm{~m}$ de largo, $1 \mathrm{~m}$ de profundidad y $0.8 \mathrm{~m}$ de ancho y se construyeron tres por cada árbol (a $50 \mathrm{~cm}$ del tronco, zona de goteo y la parte media entre las anteriores). En cada tipo de suelo se emplearon cuatro árboles homogéneos los cuales tenían 12 años de edad en suelo arenoso y 9 años de edad en el arcilloso. Las raíces se agruparon en tres estratos de profundidad (de 0 a $30 \mathrm{~cm} ; 30-60 \mathrm{~cm}$ y de $60-100 \mathrm{~cm}$ ). Se analizó el número de raíces con el programa SAS considerando a la profundidad y a la distancia como factores. La mayor cantidad de raíces se presentó, para ambos tipos de suelo, en el estrato de 0-30 cm de profundidad decreciendo hacia los estratos más profundos. Se observó la tendencia en el número de raíces, a descender conforme se incrementa la distancia desde el tronco. Las raíces más numerosas fueron las R4 o fibrosas seguidas en orden decreciente por R3, R2 y R1.
\end{abstract}

PALABRAS CLAVE ADICIONALES: distribución radical, diámetro, profundidad del suelo.

\section{DISTRIBUCIÓNRADICAL DE ÁRBOLES DE LIMÓN PERSA(Citrus latifolia Tan.)}

\begin{abstract}
This study was done with the aim of investigate on the root distribution of persian lime trees growing in a sandy soil and on a clay one in Martínez de la Torre, Ver. It was employed the soil trench profile method, and the roots were classified into four categories, according to their diameter: R1 (>15 mm), R2 (5-15 mm), R3 (1-5 mm) and R4 (<1 mm). The trenches were $4 \mathrm{~m}$ long, $1 \mathrm{~m}$ deep and 0.8 $\mathrm{m}$ wide and three trenches were made for each tree $(50 \mathrm{~cm}$ from the trunk, canopy perimeter and half-way between them). For each soil four homogeneous trees, twelve-years-old and nine-years-old for the sandy soil and for the clay one, respectively. The roots were grouped into three depth stratum $(0-30 \mathrm{~cm}, 30-60 \mathrm{~cm}$ and $60-100 \mathrm{~cm})$. The number of roots was submitted to an analysis of variance and to a multiple means comparition test, considering the depth and the distance from the trunk as factors. The higher number of roots was observed, for both types of soil, in the $0-30 \mathrm{~cm}$ deep soil stratus, decreasing in the deeper strata. The number of roots followed the tendency to decrease as the distance from the trunk increased. The most abundant roots were R4, or fibrous, followed by R3, R2 and R1.
\end{abstract}

ADDITIONAL KEY WORDS: root distribution, diameter, soil depth.

\section{INTRODUCCIÓN}

Para el año 2004, la superficie total de cítricos en México fue de 657,000 ha, de las cuales 598,000 se encontraban en producción (SAGARPA, 2004). Para el cultivo del limón persa se reporta a nivel nacional para el 2004 una superficie de 47,292 ha plantadas, generando 642,429 t anuales con un valor de $\$ 1,030$ millones, siendo el rendimiento promedio de $14.2 \mathrm{t} \cdot \mathrm{ha}^{-1}$. La importancia de 
este cultivo radica en que se exporta hasta el $80 \%$ de su producción, principalmente a los Estados Unidos. El estado de Veracruz ocupa el primer lugar nacional en cuanto a volumen de producción de este cultivo, reportándose para el año 2004 una superficie de 25,659 ha en producción, generando 381,199 t anuales con un valor de $\$ 637$ millones, siendo el rendimiento promedio estatal de $14.8 \mathrm{t} \cdot \mathrm{ha}^{-1}$; destaca en cuanto a su producción la región de Martínez de la Torre, Ver. (SAGARPA, 2004). Además de las divisas que produce, genera más de 2.5 millones de jornales anuales durante los procesos de producción, cosecha, empaque y comercialización (Curti et al., 2000).

En México, el principal portainjerto para la producción de limón persa, en más del $90 \%$ de las plantaciones, es el naranjo agrio (Citrus aurantium L.) debido a sus características (Curti et al., 2000).

Estudios sobre la distribución del sistema radical de los cítricos muestran el efecto que ejercen las propiedades físico-químicas, el manejo del suelo y del cultivo, sobre el desarrollo y disposición de las raíces (Avilán et al., 1979; Avilán et al., 1982; Avilán et al., 1983; Avilán et al., 1984; Castle, 1978; Ford, 1954; Hagin et al., 1965; Morín, 1980; Weir, 1976).

Avilán et al. (1986), comparando la distribución radical de naranjo agrio, mandarino cleopatra y limón volkameriano observaron que las diferentes texturas en el perfil del suelo tienen una gran influencia sobre la distribución de las raíces. Otros estudios muestran diferencias en la distribución y peso radical en suelos de textura gruesa y fina (Avilán et al., 1979; Hagin et al., 1965). Castle y Youtsfy (1977) indican que en suelo de textura arenosa, el naranjo agrio presentó un vigoroso y extenso sistema radical. Respecto a lo anterior, la distribución radical en cítricos se afecta negativamente por horizontes con alto contenido de arcilla, debido a una baja permeabilidad del suelo (0.2 pulgadas $\cdot h^{-1}$ de agua o menos), lo cual afecta la cantidad de oxígeno presente en el mismo (Ford, 1973; Obreza y Collins, 2002).

De acuerdo con Noling (1992), en los horizontes del subsuelo con capas de arcilla, piedra caliza o marga (tipo de roca sedimentaria compuesta principalmente de caliza y arcilla), pH menor a 5 y altos niveles de metales (cobre, zinc y manganeso), el crecimiento radical en profundidad se restringe.

Noling (1992) y Koller (1994, citado por Vieira et al., 2004) mencionan que los cítricos en suelos arenosos exploran grandes profundidades, con más del $50 \%$ de sus raíces fibrosas hasta los $75 \mathrm{~cm}$; en suelos mal drenados la profundidad de enraizamiento se limita a $70 \mathrm{~cm}$, con $75 \%$ de raíces en la parte superficial $(0$ a $40 \mathrm{~cm})$ del suelo. La cantidad de raíces tiende a ser mayor en la parte superficial del suelo y al aumentar la profundidad, va disminuyendo gradualmente aquella.
Hardy (1974) define el espacio radical como el volumen de suelo que posee las características convenientes para el desarrollo y funcionamiento de las raíces. Conforme aumenta la compactación, el número de raíces disminuye, pero en suelos con porosidad mayor al $40 \%$ pueden penetrar sin dificultad. Machado y Coelho (2000, citados por Vieira et al., 2004), para lima persa injertada sobre lima 'Cravo', encontraron una profundidad efectiva (espacio en el que las raíces de las plantas pueden penetrar sin mayores obstáculos) de $40 \mathrm{~cm}$ para huertos no irrigados. Vieira y Gomes (1999) encontraron que la profundidad efectiva de raíces para lima 'Rangpur' injertada con lima persa fue de $50 \mathrm{~cm}$ para el caso de huertos irrigados. Respecto a la práctica de irrigación, Noling (1992) indica que afecta la distribución y desarrollo de raíces, encontrándose la mayor concentración de las tipo fibrosas dentro del volumen de suelo húmedo alrededor de los emisores.

Bryla et al. (2001) determinaron que la mayoría de las raíces finas están distribuidas cerca de la superficie del suelo. Vieira et al. (2004), correlacionando la cantidad de raíces y la distancia desde el tronco, en portainjertos para limón persa en suelo arcilloso, justifican la fertilización y encalado al colocar 2/3 del fertilizante en banda dentro de la proyección de la copa y $1 / 3$ fuera de la misma, ya que ahí se ubica la mayor cantidad de raíces.

Noling (1992) menciona que la más alta densidad de raíces en los primeros $60 \mathrm{~cm}$ de la superficie del suelo se ubica bajo la copa del árbol, disminuyendo conforme se incrementa la distancia desde el tronco hacia la zona de goteo. Asimismo, indica que la temperatura mínima del suelo para el crecimiento de raíces de cítricos es de $12.2^{\circ} \mathrm{C}$, con el período más activo en verano $\left(26.6^{\circ} \mathrm{C}\right)$.

Con relación a la distancia que exploran las raíces desde el tronco hacia la zona de goteo (distancia lateral), de seis portainjertos, Vieira et al. (2004) encontraron que aquella fue similar al radio de la copa del árbol, presentándose mayor concentración de raíces bajo la proyección de la copa; sin embargo, Machado y Coelho (2000, citados por Vieira et al., 2004) encontraron distancias laterales más pequeñas que el radio de la copa para el portainjerto lima 'Rangpur'.

Quintero et al. (1997a), estimando la biomasa y distribución radical en naranja 'Valencia Tardía' injertada sobre naranjo agrio, encontraron que la mayor cantidad de raíces se localiza en los primeros $60 \mathrm{~cm}$ de profundidad. Quintero et al. (1997b) encontraron que la mayor cantidad de raíces se localiza después de los $100 \mathrm{~cm}$ de longitud a partir del tallo hacia la zona de goteo, y dentro de los primeros $60 \mathrm{~cm}$ de profundidad, disminuyendo la cantidad de raíces con la profundidad.

Por su parte, Mattos et al. (2003), para naranja 'Hamlin', encontraron que conforme se incrementa la profundidad y la distancia desde el tronco, disminuye la densidad de raíces, 
concentrándose las raíces fibrosas ( $<0.2 \mathrm{~cm}$ de diámetro) en los primeros $15 \mathrm{~cm}$ de profundidad, y más del $70 \%$ del total de raíces en el estrato de 0 a $45 \mathrm{~cm}$.

Jacob y Uexkull (1964), Obreza y Collins (2002) y Serpa (1969) señalan que suelos profundos, arenosos, bien aireados, sin excesos de humedad, sin capas impermeables y buena fertilidad natural, son los más apropiados para cítricos, ya que permiten un buen desarrollo radical.

Debido a que en nuestro país existen pocas investigaciones respecto a la distribución radical del naranjo agrio y la importancia que tiene el conocimiento de ésta, respecto al cultivo, en la toma de decisiones relacionadas con prácticas de manejo como la colocación y cálculo de dosis de fertilizantes, uso de maquinaria y equipo agrícola, diseño e instalación de sistemas de riego por gravedad y presurizados, así como el encalado, entre otras, el objetivo del presente trabajo fue determinar la distribución horizontal del sistema radical de árboles de limón persa injertados sobre naranjo agrio en dos tipos de suelo.

\section{MATERIALES Y MÉTODOS}

El trabajo se realizó en dos huertos de árboles de limón persa injertados sobre naranjo agrio (Citrus aurantium $\mathrm{L}$ ). El huerto 1 se ubicó en un suelo arenoso, en la población de 'La Palma', situada a $15 \mathrm{~km}$ al norte de Martínez de la Torre, Ver., a una altitud de $151 \mathrm{~m}$. El clima es cálido húmedo con lluvias todo el año, influencia de monzón, alta oscilación térmica, y una precipitación anual de $1,743 \mathrm{~mm}$ y la temperatura media anual es de $24{ }^{\circ} \mathrm{C}$ (García, 1981).

El huerto 2 se ubicó en un suelo arcilloso, en la población de 'La Peña', municipio de Nautla, Ver., a una altitud de $10 \mathrm{~m}$ y a $40 \mathrm{~km}$ al este de Martínez de la Torre, Ver. El clima es cálido húmedo, con una temperatura media anual de $25.5^{\circ} \mathrm{C}$ y una precipitación media anual de 1,338 mm (García, 1981).

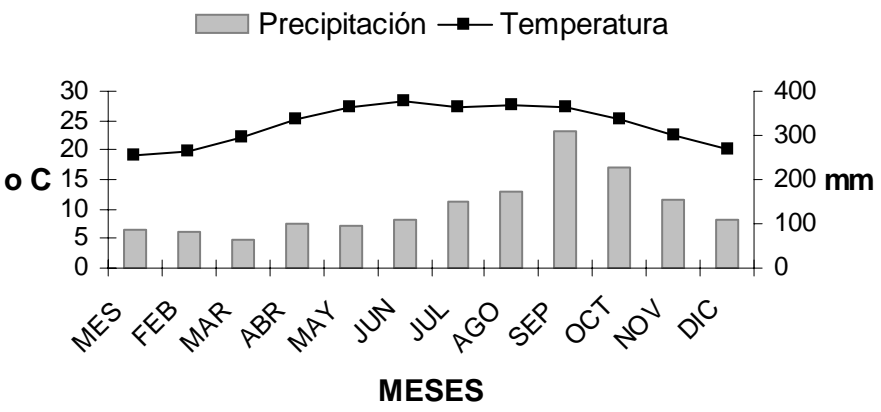

FIGURA 1. Climograma de la estación meteorológica 30102. Martínez de la Torre, Veracruz.
En el huerto 1 se emplearon árboles de 12 años de edad plantados a $6 \times 6 \mathrm{~m}$ en marco real. Para el huerto $2 \mathrm{se}$ emplearon árboles de nueve años de edad plantados a $5 \times 5$ $\mathrm{m}$ en marco real. Se seleccionaron cuatro árboles similares por cada huerto, en edad, porte, vigor, sanidad, perímetro del tronco, altura del árbol, diámetro de copa y carga de fruta.

El manejo agronómico de ambos huertos se considera normal. Para ambos huertos se realizan dos controles al año de maleza con herbicidas (glifosato), no se usa maquinaria (rastra o chapeadora) y se realizan dos fertilizaciones al año, la primera de febrero-marzo y la segunda de julio-agosto (inicio de lluvias), empleando $2 \mathrm{~kg}$ de urea al suelo por árbol. El control de plagas y enfermedades se basa en la aplicación de fungicidas (oxicloruro de cobre) para el control de la antracnosis (Colletotrichum acutatum) en floración, y acaricidas (etión) para controlar araña roja (Phyllocoptruta oleivora) y ácaro blanco (Polyphagotarsonemus latus), durante el amarre y crecimiento del fruto. Respecto a la poda, se realiza una vez al año. En el caso del huerto 1 se aplicaron a fines del mes de octubre de 2002 sustancias defoliantes (tidiazurón a $300 \mathrm{mg} \cdot$ litro-1) $^{-1}$ para incrementar la producción invernal.

El método de estudio empleado para determinar la distribución de las raíces fue el de la pared del perfil del suelo (descrito por Schuurman y Goedewaagen, 1965; Böhm, 1979). Para el huerto 1, el trabajo se inició en el mes de febrero del 2003 y para el huerto 2, en el mes julio del 2003. Se construyeron tres cepas o trincheras por cada árbol estudiado, en sentido transversal a la línea de árboles, de $4 \mathrm{~m}$ de largo, $1 \mathrm{~m}$ de profundidad y $0.8 \mathrm{~m}$ de ancho. Éstas se ubicaron a $50 \mathrm{~cm}$ del tronco del árbol, otra en la zona de goteo y otra más en la parte media entre el tronco y la zona de goteo.

Para el conteo de raíces, éstas se clasificaron, de acuerdo a su diámetro, en cuatro categorías, siendo R1 (mayores a $15 \mathrm{~mm}$ ), R2 (de 5 a $15 \mathrm{~mm}$ ), R3 (de 1 a $5 \mathrm{~mm}$ ) y R4 (menores a $1 \mathrm{~mm}$ ). Hecha la cepa, se humedeció la pared del perfil con agua empleando una aspersora de mochila y se retiró el suelo con una brocha de cerdas finas de 2 pulgadas para dejarlas expuestas, recortándose las raíces con tijeras de podar, para después dibujar el perfil de cada raíz sobre tramos de polietileno de $1.2 \mathrm{~m}$ de altura por $4 \mathrm{~m}$ de largo para cubrir la pared del perfil del suelo, empleándose para ello plumones de tinta indeleble y un marco de madera de $1 \times 1 \mathrm{~m}$, cuadriculado con hilos a cada $10 \mathrm{~cm}$, con objeto de agrupar los conteos en tres estratos de profundidad (0-30, 30-60 y 60-100 cm). Se empleó una simbología para las categorías de raíces, siendo un punto pequeño color café para R4, un punto azul para R3, un círculo azul para R2 y un círculo azul grande y relleno para R1. Posteriormente se hizo el conteo con una cuadrícula de 1 $\mathrm{m}$ por $1 \mathrm{~m}$, dividida cada $10 \mathrm{~cm}$. 
Se analizaron muestras de hojas de ramas sin frutos, de 5 a 7 meses de edad, de la quinta posición desde el ápice hacia la base, que estuvieran sanas y sin daños, y se determinaron por análisis químicos las concentraciones de $\mathrm{N}, \mathrm{P}, \mathrm{K}, \mathrm{Ca}, \mathrm{Mg}, \mathrm{B}, \mathrm{Cu}, \mathrm{Fe}, \mathrm{Mn}$ y Zn (Alcántar y Sandoval, 1999). También se analizaron muestras de suelo en cada huerto, considerando tres profundidades $(0-30,30-60,60-$ $100 \mathrm{~cm}$ ). Se determinaron las siguientes características del suelo: la textura por el método de Bouyoucos; la materia orgánica por el método de Walkley y Black; el pH con relación 1:2 suelo-agua con potenciómetro; la conductividad eléctrica leída en el extracto para pH con el mismo aparato Hanna Modelo 99120; la capacidad de intercambio catiónico por cationes intercambiables extraídos con acetato de amonio $1 \mathrm{~N}$ y pH 7.0; el $\mathrm{N}$ por el método de Kjeldahl; el $\mathrm{P}$ por el método Bray; el K por espectrofotometría de emisión de flama; el Ca y Mg por volumetría (EDTA $0.01 \mathrm{~N}$ ); el Fe, $\mathrm{Cu}, \mathrm{Zn}$ y Mn fueron extraídos y leídos en espectrofotómetro de absorción atómica; el B fue determinado por el método de la azometina-H (Etchevers, 2001).

Con los datos de números de raíces por cada categoría establecida, perfil estudiado y por cada estrato de profundidad se concentró la información para obtener los totales, promedios y porcentajes respecto a la distribución radical. Se corrió un programa de análisis de varianza (SAS) y las respectivas pruebas de medias por el método de Tukey, considerando como factores de análisis el estrato de profundidad con tres niveles $(0-30,30-60$ y $60-100 \mathrm{~cm})$, el perfil o distancia desde el tronco a la zona de goteo con tres niveles (a $50 \mathrm{~cm}$ del tronco, zona de goteo y la parte media entre las dos anteriores) y las cuatro categorías de raíces R1, (>15 mm); R2, (de 5 a $15 \mathrm{~mm}$ ); R3, (de 1 a $5 \mathrm{~mm}$ ) y R4 ( $<1 \mathrm{~mm}$ ). Cada árbol se consideró como un bloque, siendo la variable respuesta el número de raíces por cada categoría. Se analizó cada factor por separado. El análisis de la información fue para cada huerto.

\section{RESULTADOS Y DISCUSIÓN}

El Cuadro 1 muestra que el suelo del huerto 2 presentó las mejores características físico-químicas, con un $\mathrm{pH}$ cercano al neutro, que favorece la absorción de nutrimentos, en relación al huerto 1.

Empleando la clasificación de Benton-Jones et al. (1991) y los índices de balance Kenworthy (1967) con base en los valores estándar y coeficientes de variación generados por Maldonado (1999) para limón mexicano, se muestran en el Cuadro 2 la clasificación del estado nutrimental para ambos huertos, incluyendo el orden de requerimiento nutrimental (ORN).

En el Cuadro 3, para el huerto 1, destacan las raíces fibrosas R4, encargadas principales de la absorción de agua y nutrimentos, ya que presentaron valores superiores al 96 $\%$ en su número respecto a los otros tipos estudiados, que en general presentan valores muy inferiores (R3, 2\%; R2, 1 $\%$; R1, $0.5 \%$ ); coincidiendo con lo encontrado por Castle y Youtsfy (1977) y lo señalado por Noling (1992).

En el Cuadro 4, se observa que las raíces R4 presentan los mayores valores en su número en todos los estratos de profundidad y perfiles realizados, coincidiendo con Noling (1992).

Las Figuras 2 y 3 muestran que en el estrato de profundidad de 0 a $30 \mathrm{~cm}$, sin considerar la distancia desde el tronco del árbol hacia la zona de goteo, se presentaron los mayores porcentajes de raíces totales para ambos huertos estudiados, apreciándose la tendencia a descender los valores conforme se incrementa la profundidad. Lo anterior coincide con lo señalado por Quintero et al. (1997a) y Quintero et al. (1997b).

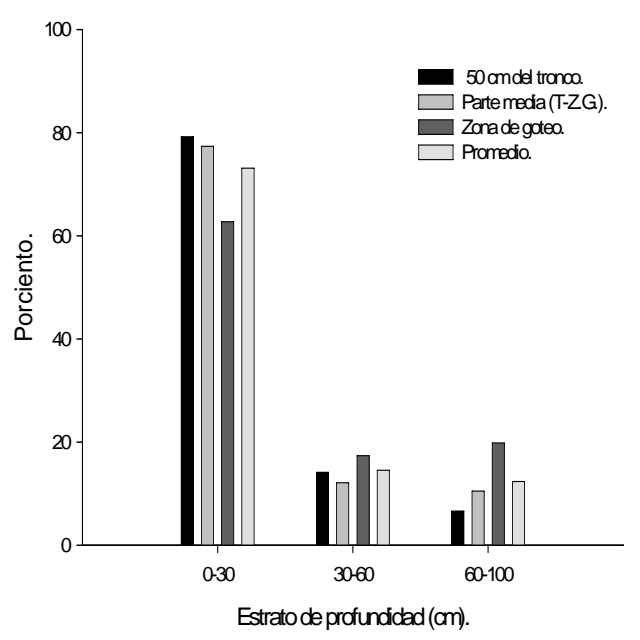

FIGURA 3. Porcentajes de raíces totales por estrato de profundidad $(\mathrm{cm})$ y por perfil realizado en Naranjo agrio. (Huerto 2).

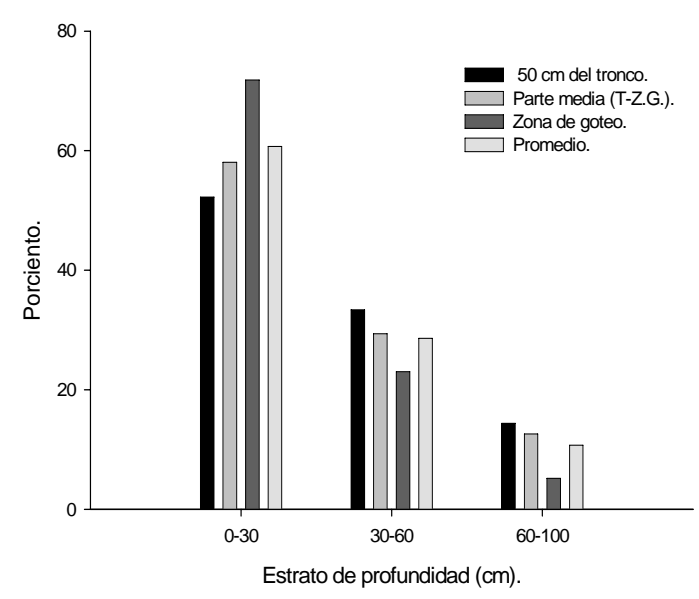

FIGURA 2. Porcentaje de raíces totales por estrato de profundidad $(\mathrm{cm})$ y por perfil realizado en Naranjo agrio (Huerto 1). 


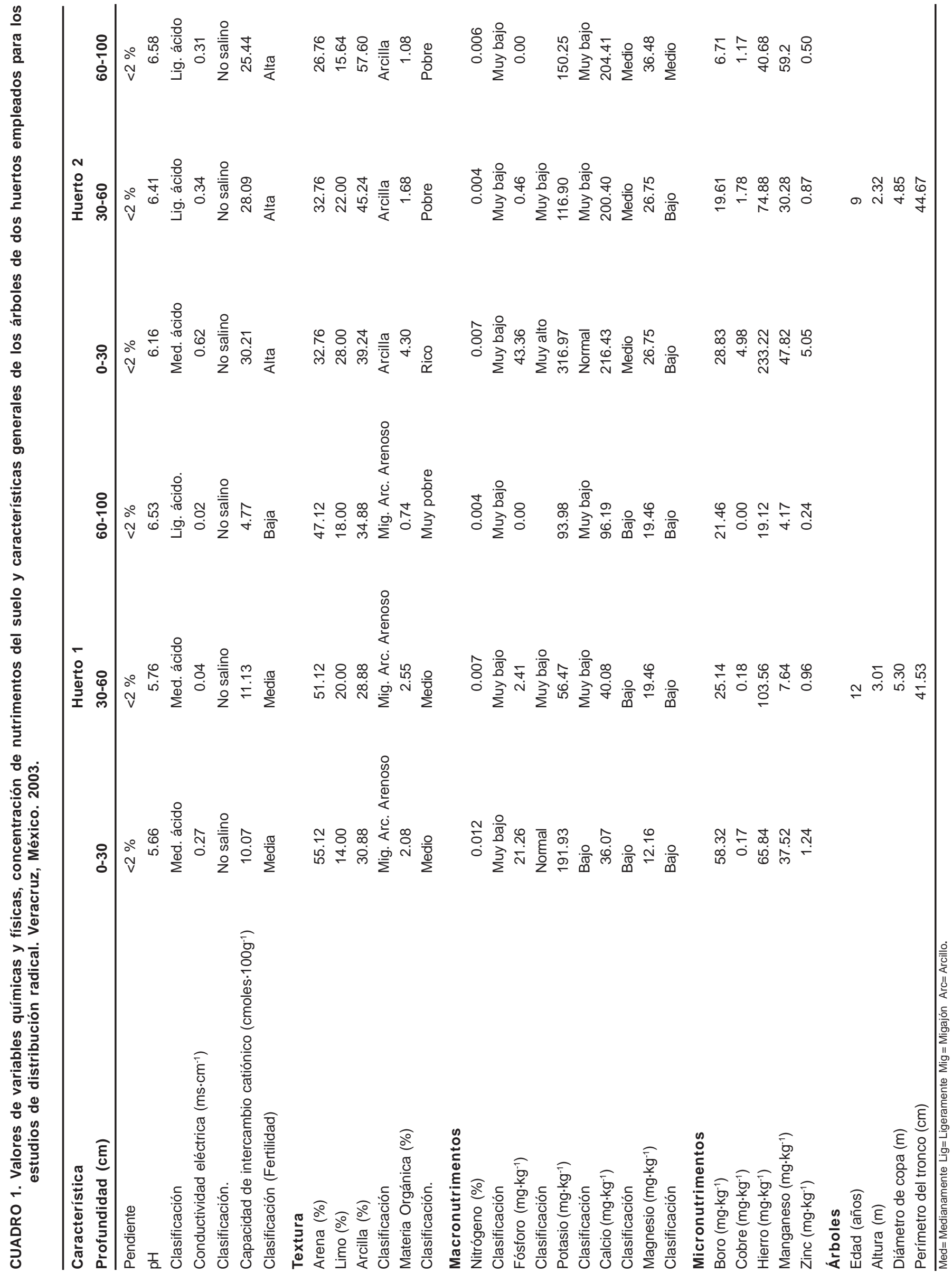


CUADRO 2. Concentración y clasificación de macro y micronutrimentos en hojas de limón persa injertado sobre naranjo agrio en dos huertos. Veracruz, México. 2003.

\begin{tabular}{|c|c|c|c|c|c|c|c|c|}
\hline Macro-Nutrimentos (\%) & \multicolumn{4}{|c|}{ Huerto 1} & \multicolumn{4}{|c|}{ Huerto 2} \\
\hline Nitrógeno & 0.579 & $\mathrm{~B}^{\mathrm{y}}$ & 35.67 & $D^{x}$ & 0.627 & $\mathrm{~B}^{\mathrm{y}}$ & 37.06 & $D^{x}$ \\
\hline Fósforo & 0.474 & $\mathrm{~S}$ & 201.95 & $\mathrm{E}$ & 0.410 & $\mathrm{~S}$ & 173.9 & $E$ \\
\hline Potasio & 1.931 & $\mathrm{~S}$ & 117.66 & AN & 1.507 & $\mathrm{~B}$ & 96.92 & $\mathrm{~N}$ \\
\hline Magnesio & 0.310 & $\mathrm{~S}$ & 76.34 & DN & 0.347 & $\mathrm{~S}$ & 81.21 & DN \\
\hline Micro-Nutrimentos $\left(\grave{ } \mathrm{g} \cdot \mathrm{g}^{-1}\right)$ & Valor & Nivel & IBK & Nivel & Valor & Nivel & IBK & Nivel \\
\hline Boro & 29.91 & $\mathrm{~B}$ & 61.38 & DN & 51.82 & $\mathrm{~S}$ & 86.2 & $\mathrm{~N}$ \\
\hline Cobre & 7.15 & $\mathrm{~S}$ & 77.67 & DN & 4.275 & B & 61 & DN \\
\hline Zinc & 17.825 & $\mathrm{~B}$ & 74.75 & $\mathrm{DN}$ & 14.025 & $\mathrm{~B}$ & 64.29 & DN \\
\hline ORN $^{w}$ & \multicolumn{3}{|c|}{$\mathrm{N}>\mathrm{Ca}>\mathrm{B}>\mathrm{Mn}>\mathrm{Zn}>\mathrm{Mg}>\mathrm{Cu}>\mathrm{Fe}>\mathrm{K}>\mathrm{P}$} & \multicolumn{5}{|c|}{$\mathrm{N}>\mathrm{Cu}>\mathrm{Zn}>\mathrm{Mn}>\mathrm{Mg}>\mathrm{Ca}>\mathrm{B}>\mathrm{K}>\mathrm{Fe}>\mathrm{P}$} \\
\hline
\end{tabular}

CUADRO 3. Distribución radical por estrato de profundidad y perfil realizado en árboles de limón persa injertados sobre naranjo agrio (Huerto 1). Martínez de la Torre, Veracruz. 2003.

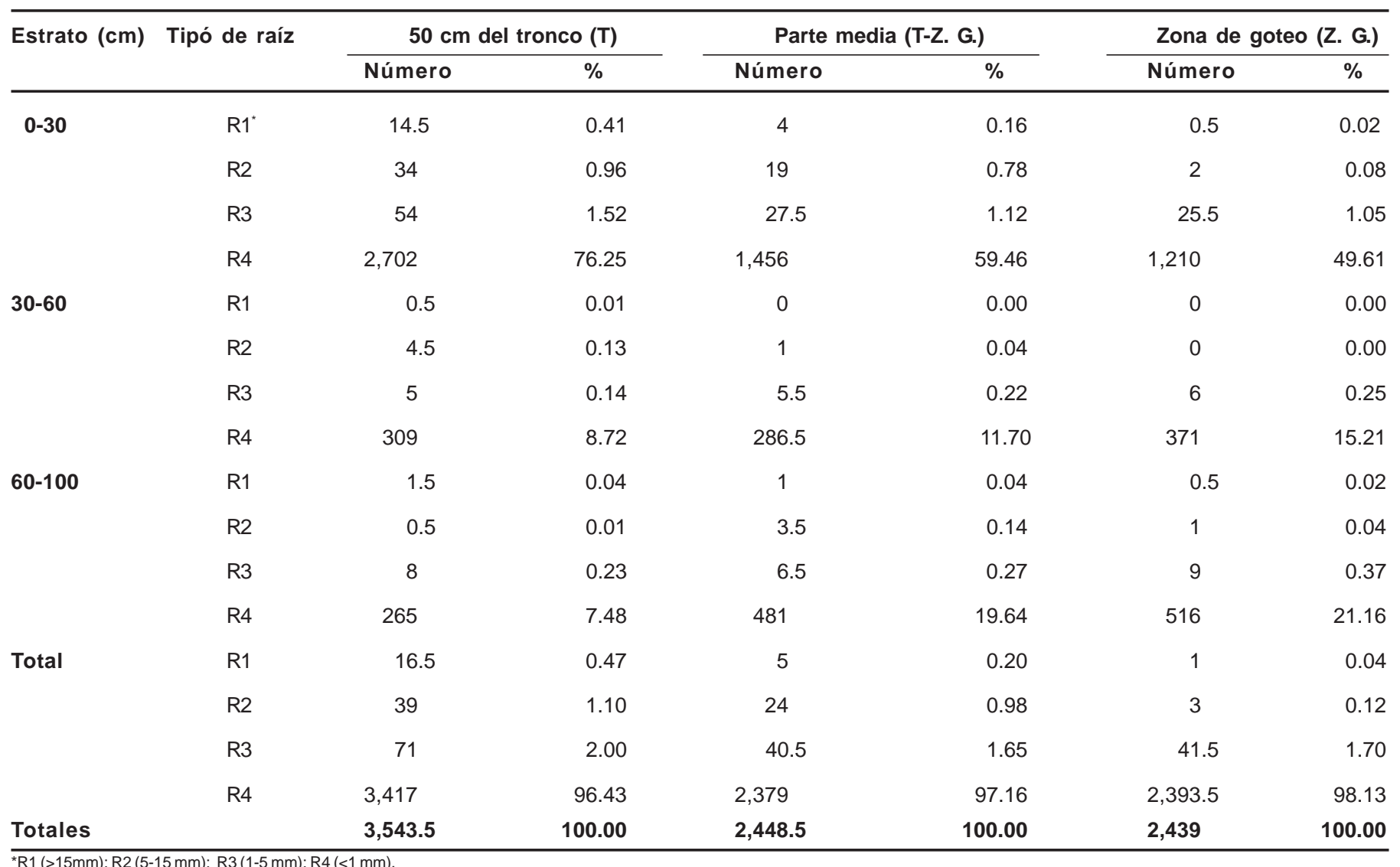


CUADRO 4. Distribución radical por estrato de profundidad y perfil realizado en árboles de limón persa injertados sobre naranjo agrio (Huerto 2). Nautla, Veracruz. 2003.

\begin{tabular}{|c|c|c|c|c|c|c|c|}
\hline \multirow{2}{*}{$\begin{array}{l}\text { Estrato } \\
(\mathrm{cm})\end{array}$} & \multirow[t]{2}{*}{ Tipo de raíz } & \multicolumn{2}{|c|}{$50 \mathrm{~cm}$ del tronco $(\mathrm{T})$} & \multicolumn{2}{|c|}{ Parte media (T.-Z. G.) } & \multicolumn{2}{|c|}{ Zona de goteo (Z.G.). } \\
\hline & & Número & $\%$ & Número & $\%$ & Número & $\%$ \\
\hline \multirow[t]{4}{*}{$0-30$} & $\mathrm{R} 1^{*}$ & 6.50 & 0.24 & 8 & 0.08 & 8 & 0.17 \\
\hline & $\mathrm{R} 2$ & 16.50 & 0.62 & 53 & 0.51 & 29 & 0.62 \\
\hline & R3 & 23.25 & 0.88 & 75 & 0.72 & 93 & 1.99 \\
\hline & R4 & $1,341.00$ & 50.48 & 5,907 & 56.74 & 3231 & 69.04 \\
\hline & $\mathrm{R} 2$ & 15.50 & 0.58 & 27.00 & 0.26 & 4.00 & 0.09 \\
\hline & R3 & 22.75 & 0.86 & 80.00 & 0.77 & 52.00 & 1.11 \\
\hline & R4 & 847.50 & 31.91 & $2,945.00$ & 28.29 & 1020.00 & 21.79 \\
\hline $60-100$ & $\mathrm{R} 1$ & 0.25 & 0.01 & 0.00 & 0.00 & 0.00 & 0.00 \\
\hline \multirow[t]{4}{*}{ Total } & $\mathrm{R} 1$ & 8 & 0.30 & 14 & 0.13 & 9 & 0.19 \\
\hline & $\mathrm{R} 2$ & 37.5 & 1.41 & 88 & 0.85 & 34 & 0.73 \\
\hline & R3 & 56.25 & 2.12 & 198 & 1.90 & 150 & 3.21 \\
\hline & R4 & 2554.5 & 96.17 & 10,111 & 97.12 & 4487 & 95.88 \\
\hline Totales & & $2,656.25$ & 100.00 & 10,411 & 100.00 & 4,680 & 100.00 \\
\hline
\end{tabular}

:R1 (>15mm), R2 (5-15 mm), R3 (1-5 mm) y R4 (<1 mm).

Los Cuadros 5 y 6 muestran que los números de las raíces $\mathrm{R} 4(<1 \mathrm{~mm})$ son los más altos y diferentes del resto, los cuales son entre sí iguales, en los dos huertos estudiados. Para ambos huertos el número de raíces en el estrato de profundidad de 0 a $30 \mathrm{~cm}$ fue mayor que en los otros estratos, mientras que los otros dos fueron iguales entre sí, coincidiendo estos resultados con lo reportado por Machado y Coelho (2000, citados por Vieira et al., 2004). El comportamiento anterior pudo deberse a que, en general, las raíces de cítricos presentan altos requerimientos de aireación, como lo señalan Stolzy et al. (1961), y por ende disminuye la cantidad de raíces hacia los estratos más profundos.
Para el efecto de la distancia desde el tronco sobre el número de total de raíces, en el huerto 1 (Cuadro 5) hubo diferencias entre los $50 \mathrm{~cm}$ del tronco y la zona de goteo. Este patrón obtenido coincide con lo señalado por Mattos et al. (2003). En el huerto 2 (Cuadro 6), no hubo diferencias entre las distancias estudiadas sobre el número de raíces totales, lo cual no coincide con lo señalado por Quintero et al. (1997b) y Noling (1992).

El Cuadro 7 muestra la comparación de las combinaciones de profundidad y distancia estudiadas para ambos huertos, observándose un comportamiento diferente en los dos huertos estudiados.

CUADRO 5. Número de raíces totales por clase y efecto de profundidad y distancia al tronco del árbol, sobre el número de raíces de árboles de limón persa en el Huerto 1. Martínez de la Torre, Veracruz. 2003.

\begin{tabular}{lrlll}
\hline Raíces & Número & Profundidad & Número & Distancia (Perfil) \\
\hline R1 $(>15 \mathrm{~mm})$ & $2.17 \mathrm{~b}^{2}$ & $\mathrm{P} 1(0-30 \mathrm{~cm})$ & $612.81 \mathrm{a}$ & D1 (50 cm del Tronco) \\
R2 $(5-15 \mathrm{~mm})$ & $8.19 \mathrm{~b}$ & $\mathrm{P} 2(30-60 \mathrm{~cm})$ & $115.44 \mathrm{~b}$ & D2 (Parte media) \\
R3 $(1-5 \mathrm{~mm})$ & $16.42 \mathrm{~b}$ & $\mathrm{P} 3(60-100 \mathrm{~cm})$ & $87.81 \mathrm{~b}$ & D3 (Zona de goteo) \\
R4 $(<1 \mathrm{~mm})$ & $1,061.31 \mathrm{a}$ & & & $173.96 \mathrm{~b}$ \\
C.V. & 129.6893 & & 129.6893 & 129.6893 \\
D.S.H. & 216.98 & 171.13 & 171.13 \\
\hline
\end{tabular}

${ }^{2}$ Valores de números con la misma letra en la misma columna son iguales de acuerdo con la prueba de Tukey a una $(P \leq 0.05)$.

R: Raíces: R1 (>15mm); R2 (5-15 mm); R3 (1-5 mm); R4 (<1 mm).

P: Profundidad (cm): P1 (0-30); P2 (30-60); P3 (60-100).

D: Distancia: D1 (50 cm del tronco); D2 (parte media); D3 (zona de goteo).

C.V.: Coeficiente de Variación.

D.S.H.: Diferencia Significativa Honesta. 
CUADRO 6. Número de raíces totales por clase y efecto de profundidad y distancia al tronco del árbol, sobre el número de raíces de árboles de limón persa en el Huerto 2. Nautla, Ver. 2003.

\begin{tabular}{lcllc}
\hline Raíces & Número & Profundidad & Número & Distancia (Perfil) \\
\hline R1 $(>15 \mathrm{~mm})$ & $1.78 \mathrm{~b}^{2}$ & P1 $(0-30 \mathrm{~cm})$ & $381.54 \mathrm{a}$ & D1 (50 cm del Tronco) \\
R2 $(5-15 \mathrm{~mm})$ & $8.50 \mathrm{~b}$ & P2 $(30-60 \mathrm{~cm})$ & $182.50 \mathrm{~b}$ & D2 (Parte media) \\
R3 $(1-5 \mathrm{~mm})$ & $20.08 \mathrm{~b}$ & P3 $(60-100 \mathrm{~cm})$ & $69.21 \mathrm{~b}$ & D3 (Zona de goteo) \\
R4 $(<1 \mathrm{~mm})$ & $813.97 \mathrm{a}$ & & & \\
C.V. & 67.452 & & 67.452 & \\
D.S.H. & 87.573 & & 69.069 & 67.452 \\
\hline
\end{tabular}

¿Valores de números con la misma letra en la misma columna son iguales de acuerdo con la prueba de Tukey a una( $P \leq 0.05)$.

R: Raíces: R1 (>15mm); R2 (5-15 mm); R3 (1-5 mm) y R4 (<1 mm).

P: Profundidad (cm) : P1 (0-30); P2 (30-60); P3 (60-100).

D: Distancia: D1 (50 cm del tronco), D2 (parte media) y D3 (zona de goteo).

C.V.: Coeficiente de Variación.

D.S.H.: Diferencia Significativa Honesta.

CUADRO 7. Resultados del análisis estadístico para el número de raíces totales en las combinaciones de profundidad y distancia en limón persa (Huerto 1 y Huerto 2). 2003.

\begin{tabular}{|c|c|c|c|}
\hline \multicolumn{2}{|l|}{ Huerto 1} & \multicolumn{2}{|l|}{ Huerto 2} \\
\hline Combinación PD & Número & Combinación PD & Número \\
\hline P1D1 & $876.0 \mathrm{a}^{\mathrm{z}}$ & P1D3 & $420.13 \mathrm{a}$ \\
\hline P1D2 & $634.9 \mathrm{ab}$ & P1D2 & $377.69 \mathrm{ab}$ \\
\hline P1D3 & $327.6 \mathrm{bc}$ & P1D1 & $346.81 \mathrm{abc}$ \\
\hline P2D1 & $156.1 \mathrm{c}$ & P2D1 & $221.75 \mathrm{bcd}$ \\
\hline P3D3 & $103.6 \mathrm{c}$ & P2D2 & $191.13 \mathrm{~cd}$ \\
\hline P2D2 & 99.5 c & P2D3 & $134.63 \mathrm{de}$ \\
\hline P2D3 & $90.7 \mathrm{c}$ & P3D1 & 95.50 de \\
\hline P3D2 & $86.2 \mathrm{c}$ & P3D2 & $81.88 \mathrm{de}$ \\
\hline P3D1 & $73.6 \mathrm{c}$ & P3D3 & $30.25 \mathrm{e}$ \\
\hline C.V. & 128.14 & & 66.081 \\
\hline D.S.H. & 390.36 & & 156.21 \\
\hline $\begin{array}{l}\text { VValores de números con la } \\
\text { Tukey a una }(P \leq 0.05) \text {. } \\
\text { P: Profundidad }(\mathrm{cm}): \mathrm{P} 1(0- \\
\text { D: Distancia: } \mathrm{D} 1(50 \mathrm{~cm} \text { del } \\
\text { C.V.: Coeficiente de Variació } \\
\text { D.S.H.: Diferencia Significa }\end{array}$ & $\begin{array}{l}\text { ma letra en la m } \\
\text { P2 (30-60); P3 } \\
\text { co); D2 (parte } \\
\text { Honesta. }\end{array}$ & $\begin{array}{l}\text { olumna son iguales de acuer } \\
\text { 0). } \\
\text {; D3 (zona de goteo). }\end{array}$ & on la prueba de \\
\hline
\end{tabular}

En el Cuadro 8 (huerto 1), destaca el tipo R4 en la profundidad de 0 a $30 \mathrm{~cm}$ que fue superior a R4 ubicadas de 30-60 cm y de 60-100 cm, y a los tipos R1, R2 y R3, que fueron iguales entre sí, en las tres profundidades estudiadas, coincidiendo con lo señalado por Bryla et al. (2001) en el sentido de que la mayoría de las raíces finas están distribuidas cerca de la superficie del suelo; se obtuvo un $75.1 \%$ (Cuadro 8) del total de raíces en el estrato de 0 a 30 $\mathrm{cm}$ de profundidad.

Respecto al efecto de la distancia (Cuadro 9) sobre el número de raíces para el huerto 1, los tipos R1, R2 y R3 fueron inferiores e iguales entre sí; mientras que los valores mayores fueron para R4 a los $50 \mathrm{~cm}$ del tronco siendo igual a R4 en la parte media y diferente a R4 en la zona de goteo, siendo este último tipo igual R4 en la parte media.

Con relación al efecto de la profundidad sobre el número de raíces, para el huerto 2 (Cuadro 10), fue supe-rior el tipo R4 en el estrato de 0 a $30 \mathrm{~cm}$; seguido por el tipo R4 ubicadas de 30 a $60 \mathrm{~cm}$ y tipo R4 ubicadas de 60 a $100 \mathrm{~cm}$ de profundidad, las cuales son diferentes entre sí. Los tipos R1, R2 y R3, en las diferentes profundidades estudiadas, fueron inferiores e iguales entre sí. Se observó la disminución del número de raíces de todos los tipos estudiados conforme se incrementó la profundidad, presentándose un $89.07 \%$ de raíces totales hasta los $60 \mathrm{~cm}$ de profundidad, coincidiendo estos resultados con los de Noling (1992), Koller (1994, citado por Vieira et al., 2004), Quintero et al. (1997a) y Mattos et al. (2003).

En general, en los dos huertos estudiados se pre-sentó el mismo patrón de comportamiento, de descender el número total de raíces conforme se incrementó la pro-fundidad del suelo, apreciándose sólo un $10.75 \%$ de raí-ces totales en el estrato de 60 a $100 \mathrm{~cm}$ para el huerto 1 (Cuadro 8) y un $10.93 \%$ de raíces totales para el mismo estrato en el huerto 2 (Cuadro 10).

Sobre el efecto de la distancia desde el tronco en el número de raíces para el huerto 2 (Cuadro 11), el tipo R4 ubicado a las tres distancias estudiadas tuvo valores que fueron iguales entre sí y superiores al resto de los ti-pos de raíces $\mathrm{R} 1, \mathrm{R} 2$ y $\mathrm{R} 3$ ubicadas en las tres distancias estudiadas. En este huerto no se presentó el mismo patrón de distribución que en el huerto 1 respecto al efecto de la distancia desde el tronco del árbol sobre el número de raíces; posiblemente esto se debió a que en la fecha en la que se efectuó el trabajo en este huerto coincide con la época de sequía (Figura 1) y a las características propias del suelo y de los árboles en estudio, ya que como se aprecia, cada tipo de raíz presentó valores iguales en las tres distancias estudiadas.

En las Figuras 4 y 5, para el huerto 1, se observan diferencias entre los números de raíces encontrados, por 
CUADRO 8. Efecto de la profundidad sobre el número de raíces, por cada tipo estudiado, en árboles de limón persa en el Huerto 1. Martínez de la Torre, Veracruz. 2003.

\begin{tabular}{|c|c|c|c|c|c|c|}
\hline Profundidad $(\mathrm{cm}) /$ Tipo de raíz & $\mathbf{R} 1$ & $\mathbf{R 2}$ & R3 & R4 & Raíces Totales & Porcentaje \\
\hline $0-30$ & $5.5 b^{z}$ & $20.2 \mathrm{~b}$ & $34.3 \mathrm{~b}$ & 2,391.3 a & $2,451.3$ & 75.1 \\
\hline $30-60$ & $0.5 \mathrm{~b}$ & $3.4 \mathrm{~b}$ & $9.8 \mathrm{~b}$ & $448.1 \mathrm{~b}$ & 461.8 & 14.15 \\
\hline Total/Tipo de raíz & 6.5 & 24.6 & 49.3 & 3,184 & $3,264.4$ & 100 \\
\hline C.V. & 128.14 & 128.14 & 128.14 & 128.14 & & \\
\hline
\end{tabular}

${ }^{2}$ Valores con la misma letra en las diferentes columnas son iguales de acuerdo con la prueba de Tukey a una $(P \leq 0.05)$.

R: Raíces: R1 (>15mm); R2 (5-15 mm); R3 (1-5 mm); R4 (<1 mm).

C.V.: Coeficiente de Variación.

D.S.H.: Diferencia Significativa Honesta.

CUADRO 9. Efecto de la distancia desde el tronco sobre el número de raíces, por cada tipo estudiado, en árboles de limón persa en el Huerto 1. Martínez de la Torre, Veracruz. 2003.

\begin{tabular}{|c|c|c|c|c|c|c|}
\hline Distancia/Tipo de Raíz & R1 & R2 & R3 & R4 & Raices Totales & Porcentaje \\
\hline Parte media (T-Z.G.) & $1.3 \mathrm{c}$ & $7.8 \mathrm{c}$ & $15.2 \mathrm{c}$ & $1,069.8 \mathrm{ab}$ & $1,094.1$ & 33.52 \\
\hline Zona de goteo & $0.4 \mathrm{c}$ & $1.8 \mathrm{c}$ & $11.5 \mathrm{c}$ & $682.2 \mathrm{~b}$ & 695.9 & 21.31 \\
\hline Total/Tipo de raíz & 6.5 & 24.7 & 49.3 & $3,183.9$ & $3,264.4$ & 100 \\
\hline
\end{tabular}

¿Valores con la misma letra en las diferentes columnas son iguales de acuerdo con la prueba de Tukey a una $(\mathrm{P} \leq 0.05)$.

R: Raíces: R1 (>15mm); R2 (5-15 mm); R3 (1-5 mm); R4 (<1 mm).

C.V.: Coeficiente de Variación.

D.S.H.: Diferencia Significativa Honesta

CUADRO 10. Efecto de la profundidad sobre el número de raíces, por cada tipo estudiado, en árboles de limón persa en el Huerto 2. Nautla, Veracruz. 2003.

\begin{tabular}{lcccccc}
\hline Profundidad $(\mathbf{c m})$ / Tipo de raíz & R1 & R2 & R3 & R4 & Raíces Totales & Porcentaje \% \\
\hline $0-30$ & $4.17 \mathrm{~d}^{\mathrm{y}}$ & $14.75 \mathrm{~d}$ & $29.50 \mathrm{~d}$ & $1,477.75 \mathrm{a}$ & $1,526.17$ & 60.25 \\
$30-60$ & $1.08 \mathrm{~d}$ & $8.08 \mathrm{~d}$ & $22.92 \mathrm{~d}$ & $697.92 \mathrm{~b}$ & 730 & 28.82 \\
$60-100$ & $0.08 \mathrm{~d}$ & $2.67 \mathrm{~d}$ & $7.83 \mathrm{~d}$ & $266.25 \mathrm{c}$ & 276.83 \\
Total/Tipo de raíz & 5.33 & 25.5 & 60.25 & $2,441.92$ & $\mathbf{2 , 5 3 3}$ & $\mathbf{1 0 0}$ \\
C.V. & 66.081 & 66.081 & 66.081 & 66.081 & & \\
D.S.H. & 190.37 & 190.37 & 190.37 & 190.37 & \\
\hline
\end{tabular}

Valores de números con la misma letra en las diferentes columnas son iguales de acuerdo con la prueba de Tukey a una $(P \leq 0.05)$.

R: Raíces: R1 (>15mm); R2 (5-15 mm); R3 (1-5 mm); R4 (<1 mm).

C.V.: Coeficiente de Variación.

D.S.H.: Diferencia Significativa Honesta.

cada tipo estudiado, para las combinaciones profundidad y distancia analizadas. Para este huerto en particular la mayor cantidad de raíces, de todos los tipos estudiados, se concentra en la profundidad de 0 a $30 \mathrm{~cm}$ a una distancia de $50 \mathrm{~cm}$ del tronco del árbol, disminuyendo conforme se incrementa la profundidad y la distancia desde el tronco hacia la zona de goteo, coincidiendo con lo reportado por Noling (1992), Koller (1994, citado por Vieira et al., 2004), Vieira et al. (2004) y Mattos et al. (2003).
Se observa que las raíces tipo R4 se concentran en mayor cantidad (Figura 5) en la superficie del suelo ( 0 a 30 $\mathrm{cm}$ ) coincidiendo con lo señalado por Bryla et al. (2001) y Avilan et al. (1979) en el sentido de que en cítricos la mayoría de las raíces finas $(<1 \mathrm{~mm}$ ) están distribuidas cerca de la superficie del suelo $(0-30 \mathrm{~cm})$.

En las Figuras 6 y 7 se observa que para todos los tipos de raíces estudiados, los mayores valores se localizan en la profundidad $1(0-30 \mathrm{~cm})$ en las tres distancias 
CUADRO 11. Efecto de la distancia desde el tronco sobre el número de raíces, por cada tipo estudiado, en árboles de limón persa en el Huerto 2. Nautla, Veracruz. 2003.

\begin{tabular}{lcccccc}
\hline Distancia/Tipo de raíz & R1 & R2 & R3 & R4 & Raíces totales & Porcentaje \\
\hline A $50 \mathrm{~cm}$ del Tronco (T) & $2.67 \mathrm{~b}$ & $12.50 \mathrm{~b}$ & $18.75 \mathrm{~b}$ & $851.50 \mathrm{a}$ & 885.42 & 34.96 \\
Parte Media (T-Z.G.) & $1.17 \mathrm{~b}$ & $7.33 \mathrm{~b}$ & $16.50 \mathrm{~b}$ & $842.58 \mathrm{a}$ & 867.58 & 34.25 \\
Zona de Goteo & $1.50 \mathrm{~b}$ & $5.67 \mathrm{~b}$ & $25.00 \mathrm{~b}$ & $747.83 \mathrm{a}$ & 780 & 30.79 \\
ITipo de raíz & 5.34 & 25.5 & 60.25 & $2,441.91$ & $\mathbf{2 , 5 3 3}$ & $\mathbf{1 0 0}$ \\
C.V. & 66.081 & 66.081 & 66.081 & 66.081 & & \\
D.S.H. & 190.37 & 190.37 & 190.37 & 20.37 & \\
\hline
\end{tabular}

zValores de números con la misma letra en las diferentes columnas son iguales de acuerdo con la prueba de Tukey a una $(P \leq 0.05)$.

R: Raíces: R1 (>15mm); R2 (5-15 mm); R3 (1-5 mm); R4 (<1 mm).

C.V.: Coeficiente de Variación.

D.S.H.: Diferencia Significativa Honesta.

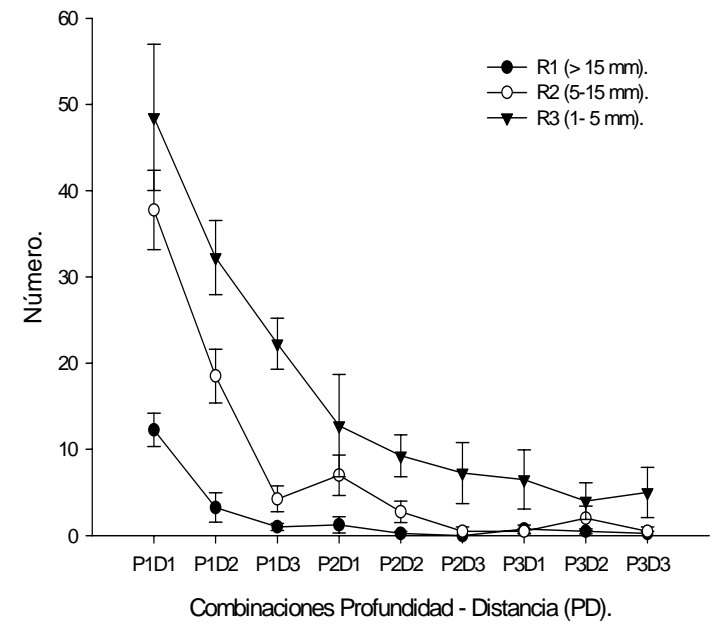

FIGURA 5. Variación del número de raíces $\mathrm{R} 4$ en las diferentes combinaciones de profundidad y distancia (Huerto 1).

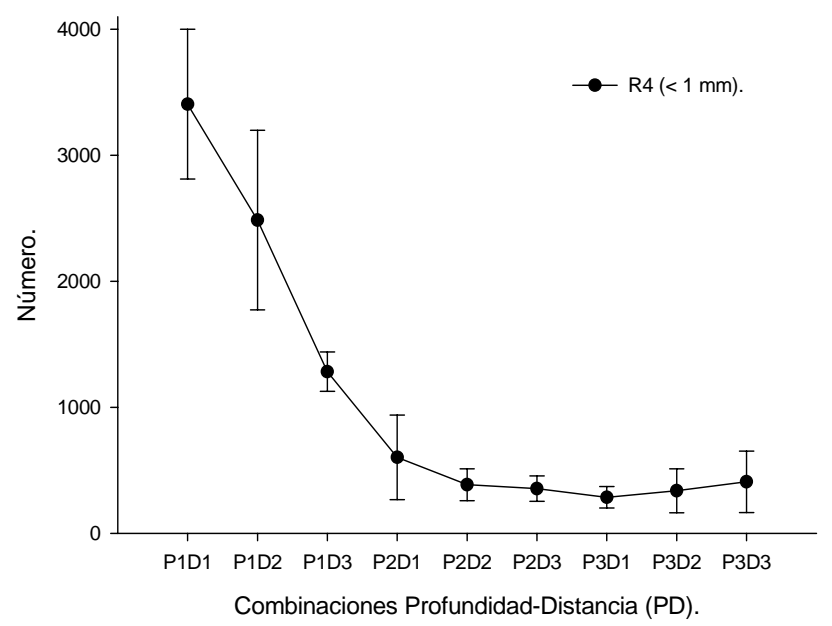

FIGURA 4. Variación del número de raíces R1, R2 y R3 en las diferentes combinaciones de profundidad $y$ distancia (Huerto 1).

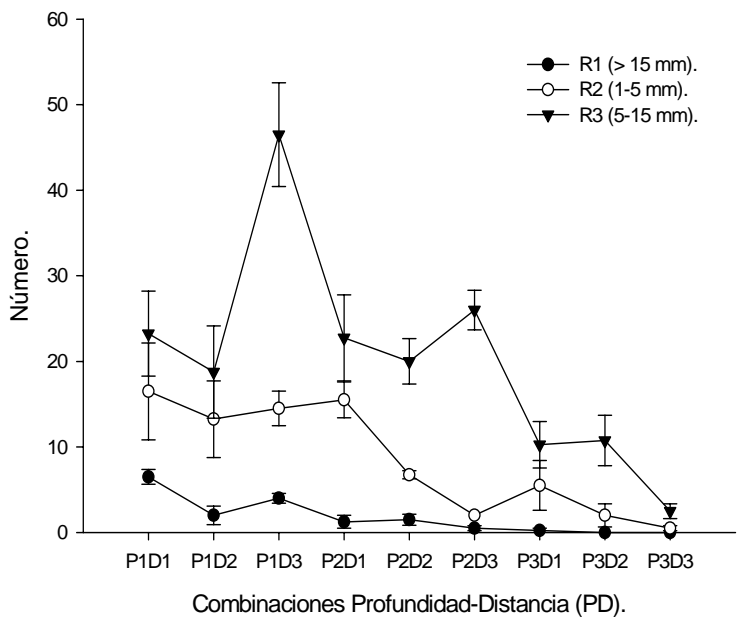

FIGURA 7. Variación en el número de raíces $R 4$ en las diferentes combinaciones de profundidad $y$ distancia (Huerto 2).

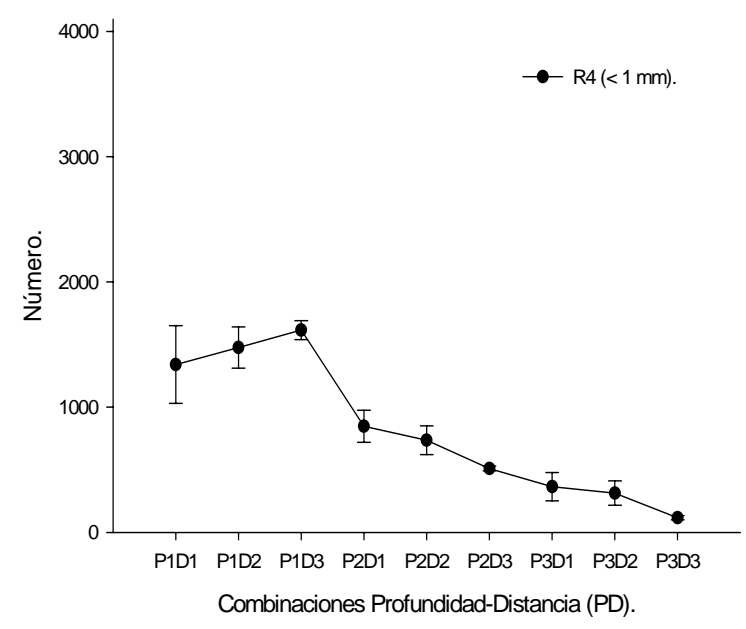

FIGURA 6. Variación en el número de raíces $\mathrm{R} 1, \mathrm{R} 2$ y $\mathrm{R} 3$ en las diferentes combinaciones de profundidad $y$ distancia (Huerto 2). 
estudiadas; sin embargo, el comportamiento es diferente al encontrado en el huerto 1, ya que no se observó el mismo patrón de distribución; y esto coincide con lo señalado por Quintero et al. (1997b), quienes encontraron que la mayor cantidad de raíces se localiza después de los $100 \mathrm{~cm}$ de longitud a partir del tallo hacia la zona de goteo, aunque, en este estudio, la época de sequía pudo haber afectado la supervivencia de las raíces.

\section{CONCLUSIONES}

Para los dos huertos la mayor cantidad de raíces se presentó en el estrato de 0 a $30 \mathrm{~cm}$ de profundidad decreciendo hacia los estratos más profundos.

Asimismo, el comportamiento observado para el huerto 1 , por parte de la cantidad de raíces respecto a la distancia desde el tronco, fue a descender conforme se incrementa la distancia desde el tronco hacia la zona de goteo. No así para el huerto 2, donde no se presentó un patrón de comportamiento bien definido.

En ambos huertos el mayor porcentaje de raíces, considerando la profundidad y la distancia desde el tronco, fue para el tipo R4 (<1 $\mathrm{mm}$ ) o fibrosas, con un promedio del $96.82 \%$, seguidas en orden decreciente por R3 (5-15 m, $2.09 \%), \mathrm{R} 2(1-5 \mathrm{~mm}, 0.87 \%)$ y R1 (>15 mm, $0.22 \%)$.

\section{LITERATURACITADA}

ALCÁNTAR, G. G.; SANDOVAL V., M. 1999. Manual de análisis químico de tejido vegetal. Publicación especial 10. Sociedad Mexicana de la Ciencia del Suelo A. C. Chapingo, México. (Faltan número de páginas)

AVILAN, L.; MENESES, L.; SUCRE, R.; PÉREZ, O.; VELARDE, C. 1979. Efectos de algunas propiedades físicas del suelo sobre la distribución radical y producción en cítricos. Agronomía Trop. 29(5): 413-427.

AVILAN, L.; LEAL, F.; MENESES, L. 1982. Distribución del sistema radical de la naranja dulce (Citrus sinensis) y grapefruit ( $C$. paradisi) sobre patrón Cajera (C. aurantium) en suelos calcáreos de la Hoya del Lago de Valencia. Agronomía Trop. 32(1-6): 155-170.

AVILAN, L.; MENESES, L.; SUCRE, R. 1983. Comportamiento del sistema radical del patrón Cleopatra injertado con naranja 'Valencia' en suelos de textura fina. Agronomía Trop. 33(1-6): 509-5 34.

AVILAN, L.; GARCÍA, MA. L.; LEAL, F.; SUCRE, R. 1984. Estudio del sistema radical del Limón Criollo (Citrus aurantifolia Swingle) en un suelo de origen aluvial. Revista Facultad de Agronomía 13(1-4): 61-72.

AVILAN, L.; VELARDE, C.;MENESES, L. 1986. Distribución del sistema radical de los patrones de cítricos naranjo agrio ( $C$. aurantium L.) Cleopatra (C. reshni Hort, ex Tan) y Volkameriana (C. volkameriana Pasq.). Agronomía Trop. 36 (4-6): 97-113.

BÖHM, W. 1979. Methods of studying root systems. Ed. Springer-Verlag. $188 \mathrm{p}$.
BRYLAD., R.; BOUMA T., J.; HARTMAOND, U.; EISSENSTAT D., M. 2001. Influence of temperature and soil drying on respiration of individual roots in citrus. Plant Cell and Enviroment 24(8): 781-790.

CASTLE, W.; KREZDORN, H. 1977. Soil water use and apparent root efficiencies of citrus trees on four rootstocks. J. Amer. Soc. Hort. Sci. (EE.UU.) 102(4): 403-406.

CASTLE, W.; YOUTSFY, CH. 1977. Root system characteristics of citrus nursery trees. Proc. Fla. State Hort. Soc. (EE.UU.) 90: 39-44.

CASTLE, W. 1978. Citrus root systems: their structure, function, growth, and relationship to tree performance. Proc. Int. Soc. Citriculture. p. 62-69.

COHEN, L. 1972. Decline of citrus true in poorly aerated soils. Proc. 18th. International Horticultural Congress. Tel-Aviv. $221 \mathrm{p}$

CURTI-DÍAZ S., A.; Loredo-Salazar, X.; Díaz-Zorrilla, U.; Sandoval R, J. A.; Hernández H., J. 2000. Tecnología para producir limón Persa. INIFAP-CIRGOC. Campo Experimental Ixtacuaco. Libro Técnico Núm. 8. Veracruz, México. 144 p.

ETCHEVERS B., J. D. 2001. Manual de procedimientos analíticos para análisis de suelos y plantas del laboratorio de fertilidad de suelos. IRENAT. Colegio de Posgraduados. Sociedad Mexicana de la Ciencia del Suelo A. C.

FORD, H. 1954. Root distribution in relation to the water table. Proc. Fla. State Hort. Soc. (EE.UU.) 67: 30-33.

FORD, H. 1973. Eight years of root injury from water table fluctuations The Citrus Industry 54(6): 10 - 16.

GARCÍA M., E. 1981. Modificaciones al sistema de clasificación climática de Köpen. Tercera edición. U. N. A. M. México. 252 p.

HAGIN, J.; LIFSHITZ, Z.; MONSELISE, P. 1965. The influence of soil aeration on the growth of citrus. Israel J. Agric. Res. (Israel) 15(2): 59-64.

HARDY, F. 1974. Root room. Trop. Agric.51(2): 272-278.

JACOB, A.; VON UEXKULL, H. 1964. Fertilización, nutrición y abonado de los cultivos tropicales y subtropicales Wageningen; Veeman 5. Zonen, Holanda, 1964. 595 p.

JONES J., B.; WOLF B.; Analysis Handbook. A practical sampling, preparation, analysis and interpretation guide. Ed. MicroMacro Publishing, Inc. USA. (Faltan número de páginas).

KENWORTHY A., L. 1967. Plant analysis and interpretation for horticulture crops. In: Plant Analysis. Hardy, G.W. (ed.) Soil Soc. Amer. Special Public. 2: 59-75.

MALDONADO T., R. 1999. El diagnóstico nutrimental en la producción de limón mexicano. Fundación Produce Michoacán y Universidad Autónoma Chapingo. México, 82 p.

MATTOS Jr. D.; GRAETZ D., A.; ALVA A., K. 2003. Biomass distribution and Nitrogen-15 partitioning in citrus trees on a sandy entisol. Soil Sci. Soc. Am. J. 67:555-563.

MORíN, Ch. 1980. Cultivo de los cítricos. Lima, Perú, Instituto Interamericano de Ciencias Agrícolas. 598 p.

NOLING J., W. 1992. Citrus root growth and soil pest management practices. Series of the Entomology and of Food and Agricultural Sciences. University of Florida. http://edis.ifas.ufl.edu.

OBREZA T., A.; Collins M., E. 2002. Common soils used for citrus production in Florida. Series of the Soil and Water Science Department University of Florida.

QUINTERO M., B.; LEE R., V.; ALCALÁ R., J. M. 1997a. Estimación de biomasa y distribución radical de naranjo 'Valencia' en suelos calcáreos de Tamaulipas. In: Memoria XXVIII 
Congreso Nacional de la Ciencia del Suelo. Villahermosa, Tabasco, México. pp 95.

QUINTERO M., B.; LEE R., V.; ALCALÁ R., J. M. 1997b. Metodologías para estudiar el sistema radical en los cítricos en Tamaulipas. In: Memoria XXVIII Congreso Nacional de la Ciencia del Suelo. Villahermosa, Tabasco, México. pp 95.

SAGARPA. 2004. Subsistema de información Agrícola. SIACON.

SCHUURMAN J., J.; GOEDEWAAGEN M., A. J. 1965. Methods for the examination of root systems and roots. Institute for soil Fertility, Groningen. Centre for agricultural publications and documentation. Holland. (Falta número de páginas).

SERPA, D. 1969. Áreas de vida de los cítricos en la región Central de Venezuela. Trabajo de Ascensos, Maracay, Universidad
Central de Venezuela, Facultad de Agronomía. 64 p.

STOLZY L., L.; SZUSKIEWICZ T., J.; LUNT, O. 1961. Root growth and diffusion rates as functions of oxygen concentration. Proceeding soil science Society American 25: 463-467.

VIEIRA D., B.; GOMES, E. M. 1999. Determinacao da profundidade efetiva do sistema radicular do limao 'Cravo' com copa de lima ácida 'Tahiti'. Laranja, 20: 419-431.

VIEIRA J., N. C. S.; MASSANORI, M. I.; COLAUTO S., N. M. 2004. Root distribution of rootstocks for 'Tahiti' lime. Sci. Agric. 61 (1): 94-99.

WEIR, C. 1976. Effect of varíous rootstock on the growth and yield of "Valencia" orange, March seedles grapefruit, and Ortanique trees in Jamaica. The Journal of Agriculture of the University of Puerto Rico 60(4): 485-490. 